\title{
Requiring physicians to dispense mifepristone: an unnecessary limit on safety and access to medical abortion
}

\author{
Wendy V. Norman MD MHSc, Judith A. Soon RPh PhD
}

$\mathrm{M}$ ifepristone, the gold standard drug for medical abortion, ${ }^{1}$ was approved by Health Canada on July 29, 2015. ${ }^{2}$ Under the federal terms of approval, physicians who wish to prescribe this treatment for their patients are required to dispense the medication themselves, which may necessitate purchase from the manufacturer, management of inventory and retail sales to patients. Health Canada's regulation bypasses the norm of drug dispensing by pharmacists, an important step in the process of ensuring medication safety. The regulation may put patients at risk and will likely limit women's access to medical abortion.

For many reasons, dispensing of mifepristone by pharmacists would be more appropriate. Pharmacists are highly trained health care professionals who provide a therapeutic doublecheck of prescribed drugs during the dispensing process. In some provinces, pharmacists initiate an additional cross-check through provincial databases for contraindications due to allergies and drugs already dispensed, and they enter data about dispensed medications into the databases. ${ }^{3}$ Surveillance through the pharmacy database can be linked to care received and to hospital admissions, which in turn allows assessment of outcomes of medical abortion, promotion of health services quality improvement and determination of distribution of and access to services. This information can inform subsequent care of women by emergency health professionals and hospitals. Pharmacists can also directly bill public and private payers for the cost of mifepristone, a service that is not available with physician-only dispensing, yet is beneficial to women. Pharmacists are well placed to dispense medications requiring exceptional vigilance and appropriate counselling - even methadone is dispensed by pharmacists. Pharmacist dispensing contributes to improved patient safety.

Physicians, on the other hand, may encounter barriers to effective dispensing. Provincial licensing bodies may explicitly prohibit physicians from selling medications and may restrict their ability to dispense. ${ }^{4}$ Physicians, who have little or no training in dispensing, may in some jurisdic- tions need to apply to become "dispensing physicians" and may need to learn and comply with the infrastructure, labelling and process standards expected of pharmacists; they must also await approval from both their provincial college of physicians and surgeons and the provincial college of pharmacists. ${ }^{4}$ Although these systems will help to ensure that physicians dispense safely, it is not apparent that this form of dispensing will confer a safety advantage over the therapeutic double-check provided through the usual physician-prescribed, pharmacist-dispensed process.

It is likely that physician-only dispensing requirements will limit women's access to mifepristone. Physicians lack the setup, time and training to manage drug inventory, including maintaining stock and ensuring that expired medicines are not released. Few doctors are likely to be willing to stock this expensive medication, reported by the manufacturer to cost $\$ 300$ per dose. ${ }^{5}$ Physicians' offices are not usually engaged in retail sales and may not have the infrastructure to sell a medication, if sales are needed to dispense it. Conflict-of-interest considerations may arise when a physician is aware that the stock purchased is near expiry, and yet is required to counsel a woman who is considering the choice between medical and surgical abortion.

It is unclear why physician dispensing is required solely for this drug. The inherent safe dispensing processes and pharmacist expertise within the Canadian health care system have advanced substantially since mifepristone was introduced in France more than a quarter century

\section{- KEY POINTS}

- Health Canada's requirement for physician-only dispensing of mifepristone is likely to reduce the safety of and access to abortion.

- Physician-only dispensing could necessitate that doctors obtain special dispensing training and authorization to order, stock, manage and sell mifepristone; these obstacles are likely to limit access to medical abortions in rural and remote locations and to place physicians in a position of conflict of interest.

- Pharmacists are trained to manage drug inventory, double check for contraindications and drug interactions through provincial databases, and directly bill public and private payers for the cost of mifepristone, all of which are services beneficial to women. 
ago. ${ }^{6}$ Although Health Canada has claimed that physician-only dispensing increases patient safety, it is difficult to see how this can be so. ${ }^{2}$ Health Canada explains that the French company that initiated the Canadian application for mifepristone approval requested physician-only dispensing as a "restrictive distribution and administration program" safety measure. ${ }^{7}$ Health Canada argues that this approach will minimize incorrect drug intake and associated health risks, and will support the product's efficacy. ${ }^{7}$ However, there is no information available on whether the applicant or the reviewers were aware that current Canadian advances in dispensing safety are in conflict with the applicant's proposal. Furthermore, pharmacist dispensing is the usual process in Canada to ensure correct drug intake and to minimize associated health risks, and it is used with drugs far more dangerous than mifepristone. Is it the prerogative of foreign corporations to design Canadian health care delivery processes?

In the United States, where physician-only dispensing is also required, medical abortion accounted for only $10 \%$ of abortions after 10 years of mifepristone availability. ${ }^{8}$ Today, 15 years after approval, mifepristone abortion in the US is still largely provided only in purpose-specific clinics concurrently offering surgical abortion.

Safe, accessible medical abortion, enabled through pharmacist-dispensed mifepristone, could reduce surgical abortions and operating room wait times and improve access to abortion. In Australia, which has safely and effectively implemented pharmacist dispensing of mifepristone, improved access to medical abortion service has been documented. ${ }^{9}$ Where mifepristone is readily accessible, women have shown a strong preference for this technique: in some countries, as many as $80 \%$ of abortions are performed with mifepristone, which reduces the need for surgery and operating room resources. ${ }^{10}$

Canada needs pharmacist dispensing for mifepristone. In this country, $96 \%$ of abortions are provided surgically, mostly in large cities at purpose-specific clinics. ${ }^{11}$ Medical abortion with pharmacist-dispensed mifepristone has the potential to diminish the disparity between rural and urban settings, through in-person visits with a woman's usual physician and through telemedicine connecting women with remote physicians. Yet with the current requirements, rural areas and areas served primarily by locum physicians, who are less able to comply with physician-dispensing approvals, training and infrastructure, will be further disadvantaged. Physician-only dispensing of mifepristone is likely to ensure the entrenchment of current inequitable access to services, thereby restricting choices and pregnancy options for women in Canada.

The Canadian government has stated that it will base policy on evidence and that it will ensure access to abortion for women throughout the country. Canadian pharmacists are the appropriate professionals to provide safe dispensing of mifepristone. Physician-only dispensing will limit the availability of mifepristone, introduce a conflict of professional interest and, in many cases, deny access to abortion for women and communities where it is needed most. We call on the federal government to act now to ensure equal, safe access to abortion for women throughout Canada.

\section{References}

1. Costescu D, Guilbert E, Bernardin J, et al. Medical abortion [SOGC clinical practice guideline 332]. J Obstet Gynaecol Can 2016;38:366-89.

2. Regulatory decision summary: Mifegymiso. Ottawa: Health Canada; 2015 [modified 2016 Jan. 13]. Available: http://hc-sc.gc. ca/dhp-mps/prodpharma/rds-sdr/drug-med/rds_sdr_mifegymiso _160063-eng.php (accessed 2016 Aug. 19).

3. Health Professions Act - bylaws. Part 1: Community pharmacy standards of practice. Vancouver: College of Pharmacists of British Columbia; 2016. Available: http://library.bcpharmacists. org/6_Resources/6-1_Provincial_Legislation/5078-HPA_Bylaws Community.pdf (accessed 2016 Aug. 19).

4. Professional standards and guidelines: dispensing and sale of pharmaceuticals by physicians. Vancouver: College of Physicians and Surgeons of British Columbia; 2009. Available: https://www. cpsbc.ca/files/pdf/PSG-Dispensing-and-Sale-of-Pharmaceuticals -by-Physicians.pdf (accessed 2016 Aug. 19).

5. Raj A. RU-486 importer wants abortion pill restrictions lifted in Canada. Huffington Post 2016 July 22. Available: www.huffingtonpost. ca/2016/07/22/abortion-pill canada_n_11146338.html (accessed 2016 Aug. 24).

6. Ulmann A, Silvestre L. RU486: the French experience. Hum Reprod 1994;9(Suppl 1):126-30.

7. Mifegymiso: summary basis of decision (SBD). Ottawa: Health Canada; 2016 [modified 2016 Jan. 11]. Available: http://hc-sc. gc.ca/dhp-mps/prodpharma/sbd-smd/drug-med/sbd-smd-2016 -mifegymiso-160063-eng.php (accessed 2016 Aug. 19).

8. Templeton A, Grimes DA. A request for abortion. $N$ Engl $J$ Med 2011;365:2198-204.

9. Grossman D, Goldstone P. Mifepristone by prescription: a dream in the United States but reality in Australia. Contraception 2015;92:186-9.

10. Løkeland M, Iversen OE, Engeland A, et al. Medical abortion with mifepristone and home administration of misoprostol up to 63 days' gestation. Acta Obstet Gynecol Scand 2014;93:647-53.

11. Norman WV, Guilbert ER, Okpaleke C, et al. Abortion health services in Canada: results of a 2012 national survey. Can Fam Physician 2016;62:e209-17.

Affiliations: Department of Family Practice (Norman) and Faculty of Pharmaceutical Sciences (Soon), University of British Columbia, Vancouver, BC; Contraception Access Research Team (Norman, Soon), Women's Health Research Institute, BC Women's Hospital, Vancouver, BC

Contributors: This commentary was equally written by both authors. Wendy Norman conceived the idea, and both of the authors developed the first draft and contributed to revisions. Both of the authors approved the final version submitted for publication and agreed to act as guarantors of the work.

Funding: Wendy Norman is supported through grant funding from the Canadian Institutes of Health Research, the Public Health Agency of Canada and the Michael Smith Foundation for Health Research. 